\title{
Are We Overtreating Hypertensive Patients? \\ Chronobiologic vs. Sphygmomanometric Evaluation of Ambulatory Antihypertensive Therapy
}

\author{
Francesco Portaluppi, M.D., Loris Montanari, M.D., \\ Michele Ferlini, M.D., and Michele Capanna, M.D.
}

\begin{abstract}
SUMmaRY
The purpose of this prospective study was to compare two methods of evaluating antihypertensive treatment efficacy: the traditional sphygmomanometric method and automatic home recording. Thirty hypertensive patients were studied who had been on antihypertensive therapy for at least 3 months. In the first 3 weeks of study their therapy was left unchanged and their diastolic blood pressures, measured with the traditional sphygmomanometer (weekly morning visit in the outpatient clinic; three successive recordings at 5 -min intervals; subject seated), were always higher than $95 \mathrm{mmHg}$. During the following 8 weeks, the physician tried to reduce diastolic blood pressure to not greater than $90 \mathrm{mmHg}$ by changing or increasing the therapy. Before and after any modification in therapy, noninvasive, automatic home, blood pressure monitorings for 24 hours were obtained and analyzed with Halberg's method of single cosinor, but were not made available to the physician until the end of the study. Automatic recordings showed that 18 patients had already had a circadian diastolic mesor $\leq 90 \mathrm{mmHg}$ under the original drug regimen. By changing or increasing the therapy, no additional pressure decrease was seen in the home pressure recordings of the whole group of patients, whereas the sphygmomanometric diastolic pressures of 15 patients fell under $90 \mathrm{mmHg}$. Our study provides additional evidence that casual sphygmomanometric measurements can be misleading when one tries to assess the efficacy of antihypertensive therapy, and a cause of potential overtreatment.
\end{abstract}

\section{Additional Indexing Words:}

Blood pressure Sphygmomanometric measurements Blood pressure monitoring Noninvasive home recordings Chronobiology Circadian rhythms Cosinor method Hypertension Therapy

RTERIAL blood pressure shows circadian patterns both in normal and hypertensive subjects. Since rhythmic fluctuations do occur, arterial

From the Institute of Clinica Medica, University of Ferrara, Ferrara, Italy.

Address for reprints: Francesco Portaluppi, M.D., Clinica Medica, Universitá di Ferrara, via Savonarola 9, I-44100 Ferrara, Italy.

Received for publication August 15, 1988.

Accepted April 21, 1989.
} 
pressure cannot be rigorously evaluated by a single or a few measurements taken with no reference to time: it is necessary to take multiple, equidistant measurements that cover at least 24 hours. Noninvasive, intermittent, automatic recording of blood pressure, either in hospitalized or ambulatory patients, is a quite simple, harmless and reliable method for assessing blood pressure patterns in individual subjects. An additional advantage of the automatic technique is its independence from the "physician effect" that, in contrast, renders the true blood pressure unpredictable. The purpose of this prospective study was to compare two methods of evaluating antihypertensive treatment efficacy: the traditional sphygmomanometric method and automatic home recording.

\section{Materials and Methods}

We studied 30 subjects referred to the outpatient clinic of our Institute of Internal Medicine with a diagnosis of essential hypertension. They were 13 men and 17 women, $51 \pm 11$ (mean $\pm S D$; range: 37 to 66 ) ycars old. The patients had already becn on antihypertensive therapy for at least 3 months with diuretics $(n=18)$, angiotensin converting enzyme inhibitors $(n=11)$, betablockers $(n=21)$ and/or other vasodilating drugs $(n=8)$. Twelve patients were on monotherapy, whereas 16 and 2 patients received the combination of 2 and 3 different agents, respectively. The patients were seen once a week for 11 consecutive weeks in the outpatient clinic between 9 and 11 am. During the first 3 weeks, they always exhibited diastolic blood pressures higher than $95 \mathrm{mmHg}$ (patient seated; three successive recordings obtained by the physician at 5-min intervals with a traditional sphygmomanometer). After the first 3 weeks of observation, during which the therapy was left unchanged, the physician tried to reduce diastolic blood pressure to a level of not greater than $90 \mathrm{mmHg}$. In order to achieve this goal, he could modify the existing therapy and use any drug regimen. Each patient was instructed to use the blood pressure recorder in his/her home, and a 24-hour blood pressure recording was obtained at the end of the third week of observation and again when the sphygmomanometric values reached $90 \mathrm{mmHg}$. However, the results of pressure recordings were not available to the physician until the end of the study. At the end of the eleventh week of observation, a home pressure recording was obtained also in those patients who still had diastolic pressure levels higher than $90 \mathrm{mmHg}$ in the outpatient clinic.

Automatic recording of blood pressure was obtained every $30 \mathrm{~min}$ for 24 hours by a quasi-portable oscillometric instrument, model BP-203 Y, manufactured by Nippon Colin Co., Ltd. (Komaki, Japan), provided with an auto- 
matically inflatable cuff. A standard $12-23 \mathrm{~cm}$ adult cuff was used. With this equipment, cuff pressure oscillations are monitored as cuff pressure decreases, with HR and BP determined by automatic analysis of the cuff pressure readings. The transducer repeatability is $\pm 1 \mathrm{mmHg}$ and the pressure span accuracy is $\pm 2 \mathrm{mmHg}$. The measured values are recorded on paper by the built-in printer with date and time of readings. Before the study, the transducer was calibrated from 0 to $250 \mathrm{mmHg}$ with a standard manometer. The patient was connected to the monitor by a $3.6 \mathrm{~m}$ long air hose. For the patients, the only limitation in pursuing normal activities during the day of monitoring was that they could not leave home.

Blood pressure recordings were analyzed according to the "cosinor" method of Halberg') using a computerized procedure that we developed." The statistical significances of the differences between the 24-hour mesors and the office mean blood pressures were calculated using the Student's paired ttest.

\section{REsults}

The results of the study are plotted in Fig. 1. In the whole group of patients, home pressures were lower by an average $23 / 17 \pm 18 / 11 \mathrm{mmHg}$ than those measured by the physician.

Before any modification of the antihypertensive therapy was attempted, out of 30 patients who showed sphygmomanometric (casual) values of diastolic pressure higher than $95 \mathrm{mmHg}, 18$ had a home (recorded) diastolic mesor $\leq 90 \mathrm{mmHg}$ (group 1) and 12 had a recorded diastolic mesor $>90 \mathrm{mmHg}$ (group 2); the patients in group 1 were also characterized by a recorded systolic mesor $\leq 140 \mathrm{mmHg}$.

Only group 1 showed a significant difference between the casual and the recorded pressure levels, both when the therapy was left unchanged (original therapy) and after a maximum of $\mathbf{8}$ weeks of intensive therapy.

After intensive therapy, no significant, additional pressure decrease was seen in the home pressure recordings of the whole group of patients that we studied; however, sphygmomanometric diastolic values of $\leq 90 \mathrm{mmHg}$ were obtained in 10 patients of group 1 and in 5 patients of group 2 . The difference in recorded diastolic mesors between groups 1 and 2 remained significant after intensive therapy; a significant difference was still present also in the recorded systolic mesors of the 2 groups. In both groups, the intensive therapy caused a nonsignificant additional decrease of recorded diastolic mesors. 


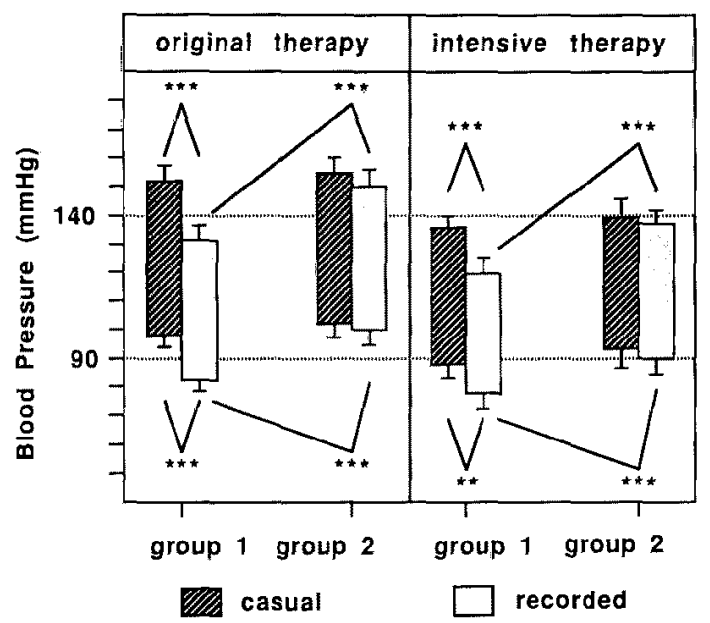

Fig. 1. Blood pressure levels in 30 hypertensive patients under standard treatment (original therapy) and after a maximum of 8 weeks of intensive therapy. Histograms represent the systolic (top margin) and the diastolic (bottom margin) levels; error bars represent the standard deviation from the mean. "Casual" represents the average value of three recordings per patient obtained by the physician in the outpatient clinic in the morning; " recorded" represents the mean mesor of the circadian rhythm calculated on noninvasive, 24-hour blood pressure recordings at home. The number of patients is 18 and 12 in group 1 and 2, respectively. Asterisks refer to the statistical difference between the 2 groups indicated by the pairs of lines. $* * * p<0.001, * * p$ $<0.005$.

\section{Discussion}

By using the "cosinor" analysis",2) it is possible to examine whether or not there is a rhythm within a 24-hour period $(p<0.05)$ and to evaluate the rhythm characteristics with $95 \%$ confidence limits. Among the rhythm parameters calculated by this statistical method, the circadian mesor (midline estimating statistic of rhythm) is equivalent to the mean 24-hour pressure. Nevertheless, the mesor is more representative of the average pressure upon the vessels' walls than the arithmetic mean when the measurements vary in density or do not cover the whole 24-hour period ${ }^{3}$; that's why we routinely use this parameter instead of the arithmetic 24-hour mean, whenever we analyze automatic blood pressure recordings. Since we analyzed 24-hour series with recordings regularly taken every $30 \mathrm{~min}$, the mesors never differed from the correspondent 24-hour mean by more than $1 \mathrm{mmHg}$. Thus, the observed differences between casual and recorded pressure levels cannot be attributed to the different method of analysis.

In the last few years a number of reservations about sphygmomanometric 
measurements of blood pressure in the physician's office have been reported. It was first noticed that blood pressures taken by a nurse are lower than those determined by a physician. ${ }^{4}$ Then, no correlation was observed, in healthy volunteers, between the mean office and the mean ambulatory blood pressure, the latter being lower by an average of $10 \mathrm{mmHg}{ }^{51}$ In another study of 249 patients with a clinical diagnosis of hypertension, more than half were normotensive when ambulatory blood pressure recordings were taken.6) In treated hypertensive patients, analyzed retrospectively, ambulatory pressures were lower by $28 / 15 \pm 23 / 13 \mathrm{mmHg}$ and $12 / 7 \pm 16 / 10 \mathrm{mmHg}$ than those measured by a physician or a nurse, respectively.? In our hypertensive group, $60 \%$ of the treated patients who still appeared to be hypertensive on the basis of clinical measurements, appeared to be normotensive when the automatic blood pressure recordings at home were analyzed.

It was also found that the blood pressure lowering effect of timolol appears to be no different from that of methyldopa in the physician's office, while in contrast, diastolic pressures recorded automatically during timolol administration are significantly lower than corresponding pressures during treatment with methyldopa ${ }^{8}$ ) In addition, the therapeutic efficacy of transdermal clonidine in hypertensive patients can appear to be negligible by using sphygmomanometric measurements in the physician's office, while ambulatory blood pressure monitoring reveals a highly significant decrease in diastolic pressure.9" Furthermore, the automatic monitoring of blood pressure is not affected by the physician-induced pressure-enhancing effect (the so-called "physician effect"), and is not influenced by a placebo effect; on the other hand, the placebo effect on sphygmomanometric pressure is poorly predictable and reproducible. ${ }^{10)-12}$

In the present study, when the office blood pressure was further reduced in those patients who still seemed to be hypertensive on the basis of clinical measurements, but who appeared to be normotensive on the basis of automatic pressure monitoring at home (group 1), no significant additional pressure decrease was seen in the home pressure recordings. These patients were found to be $60 \%$ of the total group that we studied; therefore, the change or increase in therapy was inappropriate in a majority of the hypertensive patients. The intensive treatment seemed to have less effect on home, compared to office, blood pressure. The real difference was however, since the beginning of the study, that home blood pressure levels were considerably lower than office levels. In fact, the antihypertensive effect of most drugs is maximum when severe hypertension is present, and minimum or even absent when blood pressure is normal.

Our study provides additional evidence that casual sphygmomanometric 
measurements can be misleading when we try to assess the efficacy of antihypertensive therapy. In particular, the use of the traditional sphygmomanometric method may lead to overtreatment of hypertensive patients. The aim of blood pressure control is to reduce complications of hypertension, but we still lack a clear demonstration that ambulatory blood pressure monitoring is superior in predicting the development of cardiovascular morbidity and mortality. Several cross-sectional studies have addressed this issue, ${ }^{13)-19)}$ showing that mean 24-hour or daytime blood pressure correlates more closely with the degree of target organ damage than does casual blood pressure. Two studies did include a follow-up of the patients, ${ }^{16), 19)}$ but their experimental design was neither prospective nor strictly controlled.

Altogether, it seems appropriate to stress the importance of ambulatory blood pressure recording and chronobiologic analysis in order to provide accurate and objective monitoring of antihypertensive drug effects, particularly in patients with borderline sphygmomanometric pressures, or with higher pressures but no target organ damage, where it is necessary to distinguish true from pseudo-hypertension caused by an overactive alerting reaction.

\section{REFERENCES}

1. Halberg F, Scheving LE, Lucas E, Cornelissen G, Sothern RB, Halberg E, Halberg J, Halberg F, Carter J, Straub KD, Redmond DP: Chronobiology of human blood pressure in the light of static (room restricted) automatic monitoring. Chronobiologia 11:217, 1984

2. Portaluppi F, Righetti M: Cosinor analysis of long time series on Apple II microcomputers. in Medical Informatics Europe '87, Edi Press, Roma, p 1267, 1987

3. Halberg F, Tong YL, Johnson EA: The cellular aspects of biorhythms, in Proc Symp Rhythmic Res, 8th Int Congr Anatomy, Springer, Berlin, p 20, 1967

4. Mancia G: Methods for assessing blood pressure values in humans. Hypertension 5 (suppl III) : 5,1983

5. Jacot des Combes B, Porchet M, Waeber B, Brunner HR: Ambulatory biood pressure recordings. Reproducibility and unpredictability. Hypertension 6: C110, 1984

6. Waeber B, Jacot des Combes B, Porchet M, Biollaz J, Schaller MD, Brunner HR: Ambulatory blood pressure recording to identify hypertensive patients who truly need therapy. J Chronic Dis 37: 55, 1984

7. Brunner HR, Waeber $\mathrm{B}$, Nussberger $\mathrm{J}$ : Clinical use of non-invasive ambulatory blood pressure recording. J Hypertension 3 (suppl 2): \$13, 1985

8. Rion F, Waeber B, Graf HJ, Jaussi A, Porchet M, Brunner HR: Blood pressure response to antihypertensive therapy: Ambulatory versus office blood pressure readings. J Hypertension 3: 139, 1985

9. Schaller MD, Nussberger J, Waeber B, Porchet M, Brunner HR: Transdermal clonidine in hypertensive patients: effect on office and ambulatory recorded blood pressures. JAMA 253: 233,1985

10. Gould BA, Mann S, Davies AB, Altman DG, Raftery EB: Does placebo lower blood pressure? Lancet II: 1377,1981

11. Drayer JIM, Weber MA, De Young JL, Brewer DD: Long-term BP monitoring in the evaluation of antihypertensive therapy. Arch Intern Med 143: 898, 1983 
12. Portaluppi F, Strozzi G, Degli Uberti E, Rambaldi R, Trasforini G, Margutti A, Montanari L, Fersini C, Pansini R: Does placebo lower blood pressure in hypertensive patients? A noninvasive chronobiological study. Jpn Heart J 29: 189, 1988

13. Sokolow M, Werdegar D, Kain H, Hinman A: Relationship between level of blood pressure measured casually and by portable recorders and severity of complications in essential hypertension. Circulation 34: 279, 1966

14. Floras J, Hassa MO, Sever PS, Jones JV, Osikawska B, Sleight P: Cuff and ambulatory blood pressure in subjects with essential hypertension. Lancet II: 107, 1981

15. Rowlands DB, Ireland NA, Grower DR, McLeavy AB, Stallard TJ, Littler WA: The relationships between ambulatory blood pressure and echocardiographically assessed left ventricular hypertrophy. Glin Sci 61: 101s, 1981

16. Perloff D, Sokolow M, Cowan R: The prognostic value of ambulatory blood pressure. JAMA $249: 2792,1983$

17. Devereux R, Pickering T, Harshfield GA, Kleinert HD, Denby L, Clark L, Pregibon D, Jason M, Kleiner B, Borer JS, Laragh JH: Left ventricular hypertrophy in patients with hypertension: importance of blood pressure response to regularly recurring stress. Circulation 68: 470, 1983

18. Pessina AC, Palatini P, Sperti G, Cordone L, Libardoni M, Mos L, Mormino P, Di Marco A, Dal Palu C: Evaluation of hypertension and related target organ damage by average day-time blood pressure. Clin Exp Hypertens [A] 7: 267, 1985

19. Mann S, Millar-Craig MW, Raftery EB: Superiority of 24-hour measurement of blood pressure over clinic values in determining prognosis in hypertension. Clin Exp Hypertens [A] 7: 279,1985 